\title{
Supporting Coherence Formation in Learning from Multiple Representations
}

\author{
Tina Seufert \\ University of Landau, Germany \\ Department of General and Educational Psychology \\ Thomas-Nast-Str. 44 \\ D - 76829 Landau \\ seufertt@uni-landau.de
}

\begin{abstract}
Multimedia learning environments combine multiple forms of representations like texts, static and animated pictures or graphs. Knowledge acquisition from multiple representations requires that learner create referential connections between corresponding elements and corresponding structures in different representations. As this process is usually difficult, learners frequently fail to construct coherent mental representations and, thus, do not sufficiently understand the subject matter. This paper analyzes the effects of different kinds of instructional help on the process of coherence formation from multiple representations by learners with different prior knowledge. Three groups of university students with different domain-specific knowledge had to learn a complex subject matter from chemistry using six different forms of representation. In addition, a first group received directive help for coherence formation. A second group received non-directive help, and a third group received no instructional help. Results indicate that directive help is effective for recall performance because of its summarizing and repeating function. Furthermore learners with different levels of prior knowledge show different reactions when help is given. For learners with insufficient prior knowledge help is not helpful or in case of recall performance even harmful. Learners with a medium level of prior knowledge can increase especially their comprehension performance when help is offered whereas learners with too much prior knowledge seem not to be affected from help.
\end{abstract}

\section{Theoretical background}

Multiple representations can serve many functions for learning. First, multiple representations may complement each other with regard to their content, their computational efficiency and with regard to learner characteristics and preferences. A second function is that multiple representations may constraint the interpretation of another representation (Ainsworth, 1999).

The combination of representations enables learners to deal with the material from different perspectives and with different strategies, as in the case of dynamically linked learning environments, where for example an exchange of variables in a formula is directly displayed as an exchange of graphs. The manipulation of two different representational formats supports two different ways of insight to the same learning content and may have synergetic effects on the construction of coherent knowledge structures. However, this synergy does not emerge per se. Learners must interconnect the external representations and actively construct a coherent mental representation in order to benefit from the complementing and constraining functions of multiple representations. Many studies have shown that learners often do not use multiple representations effectively (van Someren, Reimann, Boshuizen \& deJong, 1998). Especially learners with low prior knowledge often have problems with the co-ordination and integration of multiple representations (Yersuhalmy, 1991; Kozma \& Russell, 1996). They do not use different representations but rather concentrate only on one representation, often the more familiar or concrete one (Scanlon, 1998; Tabachnek \& Simon, 1998; Cox \& Brna, 1995). These learners only switch between representations in the case of problems in understanding the actually employed representation (Tabachnek \& Simon, 1998). These findings indicate that learners should get support in their coherence formation process in order to benefit from multiple representations.

As coherence formation requires that the learners create referential connections between corresponding elements and corresponding structures in different representations, the notion of structure mapping can be used as a conceptual tool to analyze this process (Gentner \& Markmann, 1997). If a learner is offered a text and a picture, he or she has to understand the information from both sources. In text comprehension, this includes searching for relevant elements and identifying the relevant relations between those elements within the text. In picture comprehension, a 
corresponding process is necessary based on pictorial information. These processes can be called intrarepresentational coherence formation. Furthermore, the learner has to find corresponding elements in the text and in the picture and has to interrelate these elements. This process can be called inter-representational coherence formation. Only if learners are able to construct such relations both within and between different representations, they can acquire a deeper understanding and as a result are able to construct coherent knowledge structures. This complex mapping process makes great demands on learners cognitive and metacognitive skills and as mentioned above a lot of studies have shown that especially individuals with low prior knowledge have problems with the coordination and integration of multiple representations (Ainsworth, 1999).

How does domain-specific prior knowledge influence the process of coherence formation? A main difference between those who are knowledgeable in a field and those who are not is a difference in perception. Novices often concentrate on surface features of the unknown representations and are not able to identify conceptually relevant entities (Lowe, 1996). In addition they seem to have problems to build up higher order information structures including complex chunks of information. As the number of chunks that can be processed simultaneously in working memory is limited, it is advantageous to build up broader chunks in order to have more information available at the same time. Availability of a high amount of information is especially important for coherence formation with different representations. Thus, a novice who is only able to store information in small chunks in working memory can be cognitively overloaded relatively quickly.

If learners with low prior knowledge are disadvantaged due to cognitive overload in knowledge acquisition from multiple representations, the question arises how these learners can be supported in their process of coherence formation. Mapping of corresponding elements between different representations can be supported by dynamiclinking or by highlighting these elements with visual cues, for example with different colours or arrows. However, this emphasis could lead to a focus on surface features, whereas higher order semantic analysis of the given representations would receive less attention. Therefore it seems more appropriate to give semantic aids, which can be provided in a more directive or in a more non-directive way.

Directive help provides explicit hints what elements and relations are relevant within each representation and which one can be mapped across the given representations. It guides learning activity directly, which may relieve learners and therefore can decrease cognitive load. Furthermore, directive help repeats elements and relations of the learning material explicitly and, therefore, leads to further encoding of the learning content. Due to the difficulty of coherence for learners with low prior knowledge, this kind of guidance might be especially helpful for this group. However, recent instructional theories emphasise that learners are not passive recipients of learning material. Instead, they actively deal with their environment. According to this view, instruction should be less directive or non-directive.

Non-directive help enables learners to discover the relevant aspects in a self-directed manner. It is more implicit in the way that learners are only prompted that there is something in one display of the material that can be mapped onto another display. Structure mapping with this kind of non-directive guidance, however, might be more demanding than with directive help.

Generally, the directivity of help for coherence formation should be adapted to the learners' prior knowledge, as was mentioned already by Wygotsky (1987) in his concept of zone of proximal development. Due to the different kinds of required cognitive processing, one can assume that the more guided kind of help is especially useful for learners with low prior knowledge, whereas the more demanding non-directive help should be more suitable for learners with high prior knowledge (McNamara, Kintsch, Songer \& Kintsch, 1996).

\section{Research Questions and Hypotheses}

Accordingly the aim of the study was to answer the general question how coherence formation in learning from multiple external representations can be supported. More specifically it was asked:

(1) Should help be provided in a more directive or in a more non-directive way?

(2) How does directive help and non-directive help respectively influence coherence formation with different levels of learners' prior knowledge?

Referred to the first question it is assumed that the directivity of help has different effects depending on the learning criterion. For recall tasks where learners have to recognize and reconstruct parts of the learning material directive help should be more effective because of its explicitness and its summarizing and repeating function. For 
comprehension performance it should be profitable to deal actively with the learning material what is supported by non-directive help. But taking into account the great demands of this help it may not surpass directive help.

Concerning the second question there are different hypotheses for learners with low, medium or high levels of prior knowledge.

It is assumed that learners with low levels of prior knowledge do not profit from help for structure mapping, neither directive nor non-directive help. The most effective help for them would be to provide additional conceptual information, which would enable them to identify relevant entities (Schwartz \& Bransford, 1998). Learners with a medium level of prior knowledge already possess this prerequisite and therefore help for structure mapping should be helpful: directive help especially for recall tasks whereas for comprehension both kinds of help may be advantegous. Learners with high levels of prior knowledge do not profit from help, because they do not need it anymore. They are able to map relevant structures within and between the given representations without further help.

\section{Method}

Subjects

Eighty-six university students of psychology and educational sciences took part in the experiment.

\section{Materials}

Pre-test Material: Students were asked to deal with different biochemical tasks in order to investigate learners prior knowledge. Additionally they were presented a series of tests in order to control for verbal and spatial abilities (Jäger \& Althoff, 1983).

Learning Material: Participants were given a computer-based learning task that dealt with the biochemical relevance of iron and vitamin $\mathrm{C}$ in human metabolism. The learning material consisted of six different graphical and verbal representations, each dealing with one facet of the phenomenon. The representations offered redundant as well as complementary information. Learners were always given two of the six displays in a fixed sequence as shown in figure 1. The groups that received either directive or non-directive help were given written supportive information in addition to the learning material. A typical example of the directive help concerning the representations shown in figure 1 is "The exchange of electrons is visible in both displays". A typical example of a non-directive help is " Are there corresponding processes in both displays and where are the differences?".

Speicherbaustein Transferrin
Transferrin bindet und transportiert Eisen
im Körper. Dabei gibt es jedoch eine
Besonderheit:
Transferrin kann Eisen nur in dreiwertiger
Form aufnehmen. Um es wieder frei-
zusetzen muß das dreiwertige Eisen
$\left(\right.$ Fe $\left.^{3+}\right)$ zu zweiwertigem Eisen $\left(\mathrm{Fe}^{2+}\right)$
reduziert werden, d.h. Fe $\mathrm{F}^{3+}$ nimmt ein
Elektron auf. In vivo geschieht das mit
Ascorbinsäure als Reduktionsmittel. In der
zweiwertigen Form ist das Eisen für
verschiedene Körperfunktionen
notwendig.

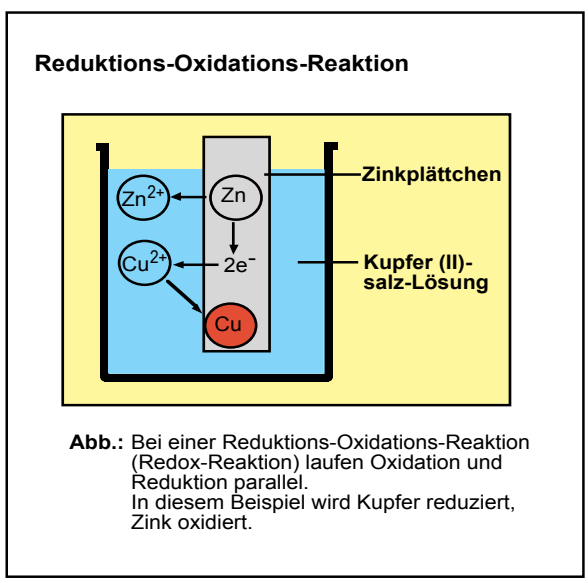

Figure 1. Example of a pair of representations used in the study

Post-test Material: Subjects were asked to carry out recognition and reconstruction tasks to assess recall performance. In addition they had to deal with a semantic network test. Subjects were given 16 important terms from the learning material and a list of possible relations between those terms and were asked to name direction and kind of relation between the given terms. For the correct relations that was explicitly mentioned in the learning material 
the subject got a score which was added to the scores from the reconstruction and recognition test. Together they form an overall recall score.

In addition subjects were asked to deal with inference and analogy tasks to analyse the degree of comprehension. The comprehension score also comprises a special score from the semantic network test, which assesses whether learners named the four central inter-relations between the given displays.

\section{Procedure and Research Design}

The study was conducted in two sessions. In the first session students were presented a series of pre-tests for prior knowledge, verbal and spatial abilities. In the second session students had to learn from the above mentioned learning material within a computer-based learning system and were afterwards asked to carry out post-tests to investigate their learning outcomes.

Subjects were randomly assigned to the three experimental groups: group with directive help, group with nondirective help and an additional control group that received no help. Prior knowledge and verbal abilities of subjects were similar in all treatment conditions.

\section{Results}

\section{(1) Does directive and non-directive help have different effects on coherence formation?}

With an alpha level of .05 , which is used for all statistical tests, the recall scores of the directive help-group (M = $16.82, \mathrm{SD}=3.99)$ are significantly superior to those of the non-directive help-group as expected $(\mathrm{M}=14.85, \mathrm{SD}=$ $4.48, \mathrm{t}(66)=1.917 ; \mathrm{p}($ one-tailed $)=.030)$. The comprehension scores of the directive and the non-directive group do not differ significantly. We expected a superiority of non-directive help but there is in fact a slight superiority of the directive help. It seems that for most of the subjects the non-directive help was too demanding.

\section{(2) How does directive help and non-directive help respectively influence coherence formation with different levels of learners' prior knowledge?}

As described above it was assumed that prior knowledge mediates the effects of help for coherence formation. Thus, three groups with different levels of prior knowledge were determined based on pre-test scores by cut-offs at the 33 rd and the 67 th percentile. The maximum score was 50 . The first group obtained an average score of $13.9(\mathrm{SD}=$ 1.4 ) and is referred to in the following as the group with low prior knowledge. The second group reached a medium level of prior knowledge with an average score of $23.5(\mathrm{SD}=4.9)$. The third group reached an average score of 33.5 $(\mathrm{SD}=6.4)$ and is referred to in the following as the group with high prior knowledge. The results for recall and comprehension will be described separately and can be seen in figure 2
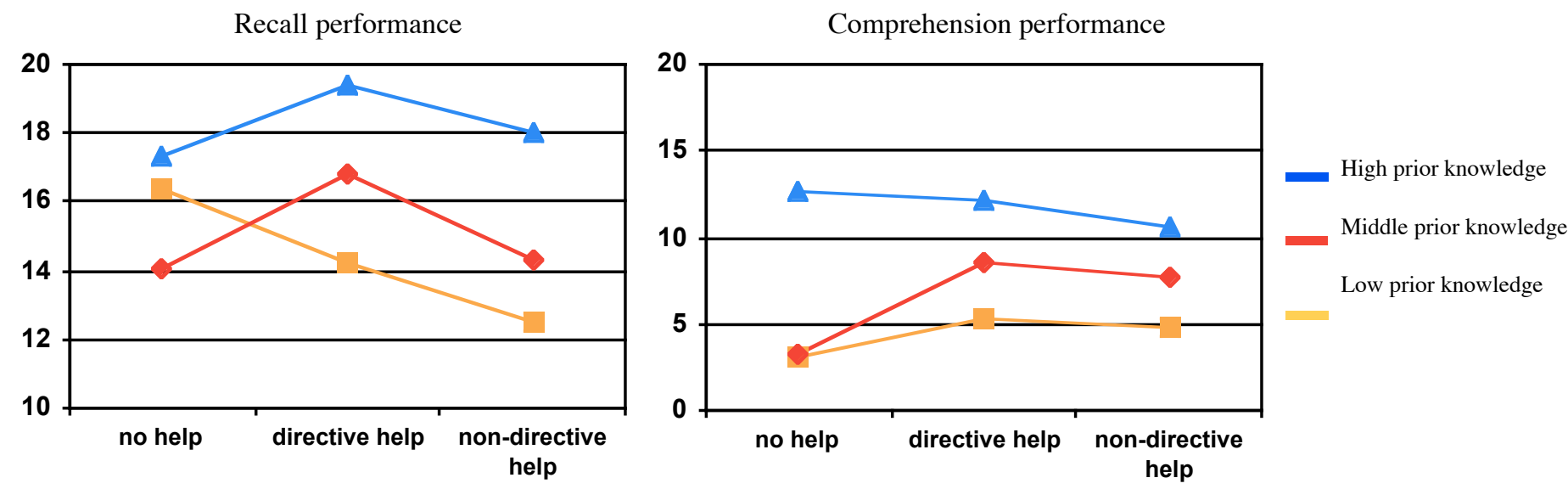

Figure 2: Recall and Comprehension Scores of learners with low, middle and high levels of prior knowledge 


\section{(a) results for recall performance}

For learners with a low level of prior knowledge help in general seems to be not really helpful or even harmful (t(27) $=1.813 ; \mathrm{p}=.081)$. Compared to the no help condition the group with directive help already shows lower recall scores but the detrimental effect is especially obvious for the non-directive help condition $(t(16)=2.344 ; \mathrm{p}=.032)$ ).

Learners with a medium level of prior knowledge seem to profit especially from directive help, which in fact turned out as the most effective help for recall. The directive help group outperforms the no help group and they also show better recall performance than the non-directive help

Although it was assumed that learners with higher levels of prior knowledge would not profit from help because they do not need it anymore they show improved recall performance under the directive help condition compared to the no help condition. They also outperformed the non-directive help group

\section{(b) results for comprehension}

The results for comprehension performance are very close to the expected pattern: As assumed, learners with low levels of prior knowledge should not profit from help because they are not yet able to use it. However, there is a tendency towards improved comprehension scores whenever help is offered (groups with help $>$ no help: $t(14,745)=$ $-1.752 ; \mathrm{p}=.100$ ). Far from significance it seems that especially directive help could be helpful for low-level learners.

As expected, learners with a medium level of prior knowledge performed significantly better in comprehension tasks when help has been offered during the learning phase (groups with help $>$ no help: $t(12,817)=-3.507 ; p=.004$ ). Compared to the no help condition learners with directive help improved their comprehension performance significantly $(\mathrm{t}(16)=2.910 ; \mathrm{p}$ (one-tailed $)=.005)$. This is also the case when non-directive help is offered $(\mathrm{t}(14,889)$ $=-2.497 ; \mathrm{p}($ one-tailed $)=.013)$ ). The two help groups do not differ significantly. Compared to learners with low levels of prior knowledge, they are obviously able to use supportive information more effectively because of their cognitive and perceptual prerequisites. According to the hypothesis that directive and non-directive help should both have advantages the scores of those groups showed no difference.

For learners with high levels of prior knowledge help seems to be not profitable because they can understand the material without further help However, help seems not to hinder comprehension processes and does not interfere with existing strategies as could be anticipated.

\section{Discussion}

The results indicate that the question how help should be provided with respect to directivity cannot be answered without taking into account the learning goal that has to be achieved. As expected directive and non-directive help seems to affect different learning processes. Directive help especially supports recall performance because of its summarizing and repeating function. Actually it was assumed that non-directive help should be particularly effective for comprehension processes because it activates learners and therefore may deepen understanding but the results do not show this tendency. In fact, directive help is also more effective for comprehension than non-directive help. As mentioned above we assess non-directive help to be cognitively very demanding. The learning material as well is very complex and demanding what could make it difficult to deal actively with the help and - at the same time - with the material. Thus it would be interesting to investigate the different kinds of structure mapping help in other, less complex domains.

Another important factor, which mediates the effects of help for coherence formation, is learners' prior knowledge. As reported above there are different reactions of learners to help depending on prior knowledge and it is possible that other learning prerequisites also have an influence on learners' coherence formation strategies and on their use of help. It is likely that comparable effects can be found for spatial as well as for verbal abilities and for cognitive styles which apply to learners' organisation of information, such as serialist / holist orientation (Pask, 1976).

As a next step a study is planned that aims to specify the sensoric channel that should be used for the presentation of help in order to reduce learners' cognitive load. As the present results show, help is not always helpful. Above all this is evident for learners with poor learning prerequisites. With reference to the great demand on learners' cognitive capacity by mapping multiple displays, one must ask if additional supportive information would lead to a cognitive overload. According to Chandler \& Sweller's cognitive load theory (1991), both the visual and the auditory working memory store have a limited capacity. Numerous studies show that when learners are engaged with visually presented material, the required effort to hold additional visually presented material within working memory can 
exceed cognitive capacity. Therefore, learning outcomes are not as good as in learning with visual material that is accompanied by auditory information (Mayer \& Moreno, 1998). Visually presented help for mapping could lead to a split attention effect in dealing with visually presented learning material. This raises the question that must be answered in subsequent studies: Which sensoric modality is the more effective to present structure mapping support visual or auditory? One must also examine the question whether split attention effects emerge to different extents for different kinds of learners.

Therefore, a research program is planned that will systematically examine the process of learning with multiple linked representations and the design and use of supportive information. Theoretically, it seems necessary to specify the interactions between external and internal representations and to discuss them in the context of current models of learning with multimedia (Mayer, 1997; Schnotz, Bannert \& Seufert, 2002). Practically, the results can provide suggestions on how to create more effective learning environments and how to effectively support learners' strategies for structure mapping in knowledge acquisition from multiple representations.

\section{References}

Ainsworth, S. (1999). The functions of multiple representations. Computers \& Education, 33, 131-152.

Chandler, P., \& Sweller, J. (1991). Cognitive load theory and the format of instruction. Cognition and Instruction, 8, 293-332.

Cox, R., \& Brna, P. (1995). Supporting the use of external representations in problem solving: The need for flexible learning environments. Journal of Artificial Intelligence in Education, 6 (2/3), 239-302.

Gentner, D., \& Markmann, A.B. (1997). Structure mapping in analogy and similarity. American Psychologist, 25, 45-56.

Jäger, A.O., \& Althoff, K. (1983). Der WILDE-Intelligenz-Test (WIT). Göttingen: Hogrefe.

Kozma, R.B., \& Russell, J. (1996). The use of linked multiple representations to understand and solve problems in chemistry. Report Oakland University.

Larkin, J.H., \& Simon, H.A. (1987). Why a diagram is (sometimes) worth ten thousands words. Cognitive Science, $11,65-99$.

Lowe, R.K. (1996). Background knowledge and the construction of a situational representation from a diagram. European Journal of Psychology of Education, 11, 377-398.

Mayer, R.E. (1997). Multimedia Learning: Are we asking the right questions? Educational Psychologist, 32, 1-19.

Mayer, R.E. \& Moreno, R. (1998). A split-attention effect in multimedia learning: Evidence for dual processing systems in working memory. Journal of Educational Psychology, 90, 312-320.

McNamara, D.S., Kintsch, E., Songer, N.B., \& Kintsch, W. (1996). Are good texts always better? Interactions of text coherence, background knowledge, and levels of understanding in learning from text. Cognition and Instruction, $14,1-43$.

Pask, G. (1976). Styles and strategies of learning. British Journal of Educational Psychology, 46, 128-148.

Scanlon, E. (1998). How beginning students use graphs of motion. In M. van Someren; P. Reimann; Boshuizen, H.P.A. \& de Jong, T. (Eds.), Learning with multiple representations (pp. 67-86). Oxford: Elsevier.

Schnotz, W., Bannert, W., \& Seufert, T. (2002). Towards an integrative view of text and picture comprehension: Visualization effects on the construction of mental models. In A. Graessser, J. Otero \& J.A. Leon (Eds.), The psychology of science text comprehension (p 385-416). Hillsdale, NJ: Erlbaum.

Schwartz, D.L., \& Bransford, J.D. (1998). A Time for Telling. Cognition and Instruction, 16, 475-522.

Tabachnek, H.J.M., \& Simon, H.A. (1998). One person, multiple representations: an analysis of a simple, realistic multiple representation learning task. In M. van Someren; P. Reimann; Boshuizen, H.P.A. \& de Jong, T. (Eds.), Learning with multiple representations (pp. 197-236). Oxford: Elsevier.

Van Someren. M.W., Reimann, P., Boshuizen, H.P.A., \& de Jong, T. (Eds.). (1998). Learning with multiple representations. Oxford: Elsevier.

Wygotski, L.S. (1987). Ausgewählte Schriften (Bd. 2). Berlin: Volk und Wissen. 
Yerushalmy, M. (1991). Student perceptions of aspects of algebraic function using multiple representation software. Journal of Computer Assisted Learning, 7, 42-57. 